\title{
Shape Change of Micelles Dragged with Constant Velocity as Addressed in Terms of Biolubrication Application
}

\author{
P. BeŁdowski ${ }^{a * *}$, R.G. WinkleR ${ }^{b}$, J. HŁadyszowski ${ }^{c}$, S. Jung ${ }^{d}$ And A. Gadomski ${ }^{a}$ \\ ${ }^{a}$ University of Science and Technology, Institute of Mathematics and Physics, Bydgoszcz, Poland \\ ${ }^{b}$ Theoretical Soft Matter and Biophysics, Institute for Advanced Simulation, \\ Forschungszentrum Jülich, Jülich, Germany \\ ${ }^{c}$ Wrocław Medical University, Department of Physical Chemistry, Wrocław, Poland \\ ${ }^{d}$ University of Science and Technology, Department of Mechanical Engineering, Bydgoszcz, Poland
}

\begin{abstract}
Molecular dynamics simulations have been performed for a model of coarse-grained lipids in water. We try to establish structural properties of micelles when they are dragged past each other. The lipid-lipid and lipid-water interactions are taken from a coarse-grained model first introduced by Marrink et al. This work focuses on shape changes upon mutual interaction of micelles when dragged with constant velocity. We find that micelles can change their shapes from spherical to cylindrical regardless of the number of constituting lipids. We address this result toward understanding facilitated vs. obstructed lubrication of articular cartilage by synovial fluid at nanoscale level.
\end{abstract}

DOI: $10.12693 /$ APhysPolA.129.188

PACS: 83.80.Rs, 47.11.Mn, 82.33.Nq, 83.50.-v

\section{Introduction and simulation method}

1.1. Preliminaries of micelles-assisted lubrication

The mechanism of articular cartilage (AC) lubrication has been studied for almost a century, but still the mechanism has remained uncovered. The problem of AC lubrication is connected to the complexity of the friction phenomena in biological systems and its multiscale effects inherent in the phenomena. AC includes two opposite rubbing surfaces and the synovial fluid (SF) between them. SF is composed mainly of water and amphiphilic components such as hyaluronic acid, surface active phospholipids (SAPL), etc. According to Gadomski [1] and refs. therein, the SAPLs in their micellar states may play a crucial role in AC lubrication [2]. Moreover, Gadomski et al. [3] suggest that the role of micelles is to lower friction by changing a sliding friction into its rolling counterpart represented - as reminiscent of bearings - by their spherical (or, rounded) shapes. The frictional behavior between soft colloids is of fundamental interest, e.g., due to the potential applications of spherical star polymers or self assembled spherical structures in drug delivery, viscosity modifications, etc. [4]. This work presents molecular dynamics simulation studies on micellar interactions in aqueous environment, where two micelles are dragged past each other with constant velocity. We employ dodecylphosphocholine (DPC) lipids for this simulation as they have natural tendency to self-assemble into micelles.

\subsection{Model}

The simulations have been performed by adopting a model by Marrink et al. [5], i.e., by a coarse-grained (CG)

*corresponding author; e-mail: piobe1000@utp.edu.pl model of water-lipid interactions. The simulation box (Fig. 1) is composed of water and DPC lipids building up

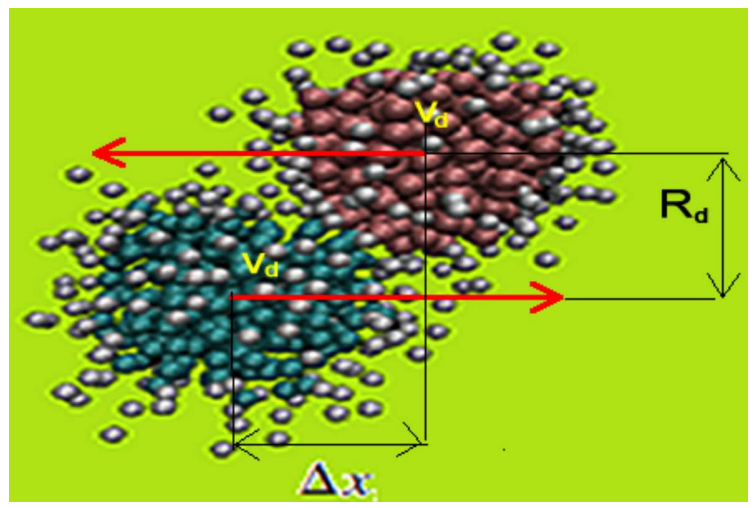

Fig. 1. Snapshot of the simulation box. Two identical micelles have been colored for better visualization (silver particles represent hydrophilic heads of lipids, whereas green and pink - hydrophobic tails). Water molecules as well as two dummy atoms (see text) are not shown in the picture. The distance between the micelles' centers of mass in $x$ - and $y$-direction are denoted as $\Delta x$ and $R_{\mathrm{d}}$, respectively. $v_{\mathrm{d}}$ stands for drift velocity of a micelle.

two micelles of either: 75, 60, 40 lipids for three different considered cases. Each lipid is composed of two parts: hydrophilic head of two CG atoms oppositely charged and hydrophobic tail composed of three apolar atoms. The interactions between each two types of atoms are described in terms of Marrink et al. model, however due to problem with stabilizing micelles cores we increased the interaction strength between tails to discourage an exchange of lipids between micelles. The Lennard-Jones (L-J) potential 


$$
V(r)=4 \epsilon\left[\left(\frac{\sigma}{r}\right)^{12}-\left(\frac{\sigma}{r}\right)^{6}\right],
$$

as well as screened Coulomb potential are used to describe pairwise interactions between atoms/ions as described in [5]. All simulations were performed using LAMMPS [6] package. SMD (steered molecular dynamics) is a method in which a centre of mass of each micelle is attached to a dummy atom by a spring which is pulled with constant velocity. The velocity is embodied in a Peclet number defined as $P e=\tau_{\mathrm{r}} / \tau_{\mathrm{d}}=\tau_{\mathrm{r}} v_{\mathrm{d}} / R_{\mathrm{g}}$, which for the presented simulations is equal to 0.25 (similarly to other soft colloids [4]). $\tau_{\mathrm{r}}$ stands for relaxation time, $v_{\mathrm{d}}$ is a drift velocity of a micelle and $R_{\mathrm{g}}$ is a radius of gyration, conforming to its standard definition. Micelles are pulled toward each other along $x$-direction and are separated in $y$-direction by $R_{\mathrm{d}} / R_{\mathrm{g}}=0.63 . R_{\mathrm{d}}$ is the distance between centers of mass in $y$-direction. The interactions between the coronas of both micelles go by employing L-J and Coulomb potentials.

\section{Results}

The simulations were performed for three different micelles' aggregation numbers, namely 40, 60, and 75 . Figure 2 shows shape changes of micelles in $y$ - and $z$-directions in terms of two ratios $R_{y} / R_{x}(\Delta x)$ and $R_{z} / R_{x}(\Delta x)$, where $R_{x}, R_{y}$ and $R_{z}$ are the corresponding axial-coordinate projections of the radius of gyration $R_{\mathrm{g}}=\sqrt{R_{x}^{2}+R_{y}^{2}+R_{z}^{2}}$. They reveal the expectation

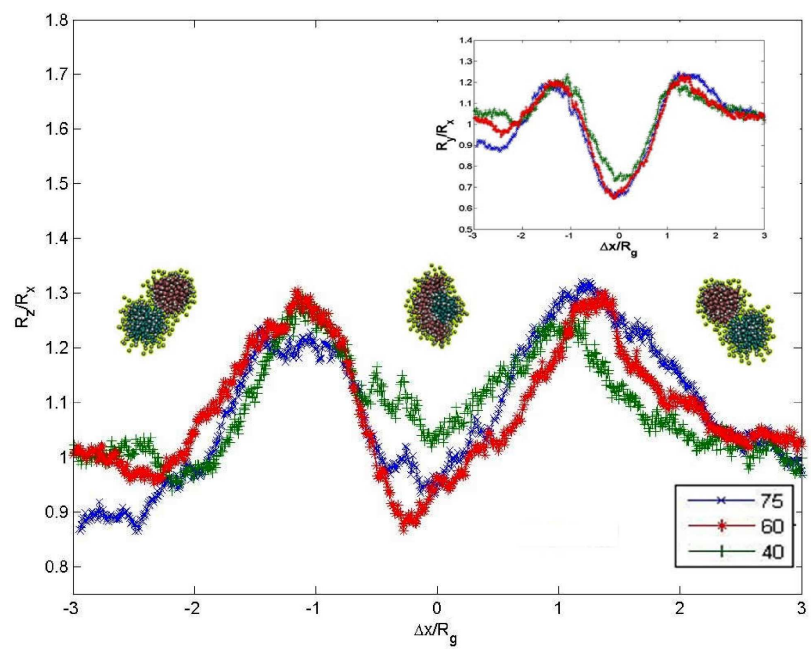

Fig. 2. Various stages of micelles' structures as a function of their centers of mass (relative) distance. Results of the different aggregation numbers (lipids per micelle): 40 (green line), 60 (red line) and 75 (blue line) are shown. The inset shows micellar isothermal shape changes in the $y$-direction. The inserted depictions of micellar bodies above their corresponding curves, reflect the occurring shape changes. that the smaller the aggregation number is the more mechanically stable the micelles can be. The micelles are changing their shapes as depicted in Fig. 2 from spherical to quasi-cylindrical or even pancake-like. The effect as such is not explicitly discussed in the present work. It, however, resembles fairly some nonequilibrium morphologies $[7,8]$, herein recoverable in terms of the aggregation number vs. drift velocity taken for different temperatures.

\section{Conclusions}

Our simulation results suggest that the morphology of two tightly interacting micelles depends sensitively on their mass center distance, entailed with the aggregation numbers. Implicitly, such complex dynamic behavior resembles qualitatively the nonequilibrium morphologies occurring in complex diffusive processes $[7,8]$. The study may also help rationalize facilitated lubrication conditions in articulating micelles-involving systems $[1,3]$. At first sight, the rationalization would rely on observing that the pancake-like structure while in motion will be more prone to deceleration as the other emergent DPC-based structures, which will make the lubrication conditions less operative [1].

\section{Acknowledgments}

This work is sponsored by UTP BS39/14. P.B. acknowledges financial support by the Erasmus program and the Forschungszentrum Jülich where this project was initiated.

\section{References}

[1] A. Gadomski, P. Bełdowski, J.M. Rubí, W. Urbaniak, W.K. Augé II, I. Santamaría-Holek, Z. Pawlak, Math. Biosci. 244, 188 (2013).

[2] Z. Pawlak, A.D. Petelska, W. Urbaniak, K.Q. Yusuf, A. Oloyede, Cell Biochem. Biophys. 65, 335 (2013).

[3] A. Gadomski, Z. Pawlak, A. Oloyede, Tribol. Lett. 30, 83 (2008).

[4] S.P. Singh, R.G. Winkler, G. Gompper, Phys. Rev. Lett. 107, 158301 (2011).

[5] S.J. Marrink, A.H. De Vries, A.E. Mark, J. Phys. Chem. B 108, 750 (2004).

[6] http://lammps.sandia.gov/.

[7] E. Brener, H. Miller-Krumbhaar, D. Temkin, T. Abel, Solid State Ion. 131, 23 (2000).

[8] A. Gadomski, Phys. Rev. E 60, 1252 (1999). 